\title{
Un vortice binario. Lingua e dialetto in Verderame di Michele Mari
}

\author{
Diego VARINI \\ Università di Parma
}

\begin{abstract}
Scopo dell'articolo è inquadrare l'architettura compositiva e linguistica del romanzo Verderame (2007) sullo sfondo della poetica di Michele Mari. La naturale vocazione al pastiche e all'intarsio delle lingue e dei dialetti tipica dello scrittore viene ricondotta all'atteggiamento fondamentalmente antiregolistico che caratterizza in primo luogo le enciclopediche frequentazioni di Mari nella sua veste di studioso accademico e di critico: a partire da una concezione dei valori letterari che privilegia costantemente l'anomalia e la stravaganza come segnali di una tensione gnoseologica sottesa alla ricerca di uno stile svincolato da qualunque inerte acquiescenza a codici e norme ricevute. In particolare, il discorso si appunta sul rapporto di esplicita contiguità che annoda le pagine di Verderame all'espressionistica mescidanza sperimentata nel Novecento italiano dalle pagine di Gadda, nel segno comune di una peculiare inserzione di elementi brutalmente dialettali entro il tessuto polifonico di una prosa aulica e vertiginosamente libresca.
\end{abstract}

Keywords: Michele Mari, Verderame, parodia, intertestualità, pastiche.

Nei termini prospettati da Harold Bloom (a partire dal celebre The Anxiety of Influence), «il desiderio di imitare i maestri coesiste col desiderio opposto di dissimularne le tracce»: in questo senso l'intertestualità funziona per Bloom sempre come «il prodotto della anxiety: tutta la letteratura nasce dal desiderio di difendersi dall'angoscia dell'influenza, dal fantasma paralizzante del Precursore» (Zatti 2016: 297). Tuttavia una simile preoccupazione sembra non avere senso per uno scrittore come Michele Mari, nella cui poetica l'idea centrale alla base della riflessione di Bloom - ovvero «la consapevolezza di muoversi dentro un orizzonte del linguaggio logorato dall'uso e risonante di echi altrui» (Zatti 20I6: 284) - non produce angustia od oppressione alcuna, perché la letteratura nasce sempre da un tentativo di emulazione rispetto ad altra e pre-esistente letteratura: moltiplicando in questo sforzo imitatativo e voluttuosamente agonistico una meravigliosa congerie di tensioni fantastiche e possibilità discorsive.

È quanto lo scrittore torna a dichiarare, per esempio, in un recente intervento che raccoglie l'esito di una conversazione tenuta in pubblico all'Università di Trento, nel 20II, e che rovescia già nella strategia del controcanto ironico sotteso al titolo (Il beneficio dell'influenza) le linee della famosa impostazione enunciata da Bloom: 
[...] l'influenza ci angoscia solo se ce ne facciamo angosciare. Per me, fin da piccolo, la confusa nozione di influenza coincideva con la percezione di essere stato tenuto a battesimo e preso per mano da tanti maestri che mi avevano insegnato e continuavano a insegnarmi di tutto: uno, un ritmo; uno, una parola strana; uno, un termine marinaro; uno, l'arte dell'ambiguità; uno, l'arte della suspense; uno, l'intreccio; uno, certe clausole. Questo ha fatto sì che quando ho cominciato a scribacchiare sentissi semmai il problema opposto: come se «fratello Michael» dovesse costruire una casa in un prato e gli altri paesani, per solidarietà, gli portassero carrettate di legname, di tubi, di chiodi, tanto da fornirgli materiale per costruire non una casa, ma un paese intero. Mi sentivo nutrito e sorretto da tutte quelle voci che confabulavano nella mia testa, la qual cosa, lungi dal paralizzarmi, mi dava un senso di fiducia e di euforia, come se mi rendesse consapevole che tutto era già incamerato e disponibile. Come scrive Borges [...] era come se, avendo dentro di sé tutti i racconti possibili, si trattasse solo di trovare le giuste combinazioni verbali, anzi alfabetiche (Mari 20I7: 728-729).

Se per un verso in Harold Bloom il rapporto fra innovazione e tradizione tende a configurarsi in forma di «scontro edipico fra un figlio e un padre (colorando la tensione liberatoria di immagini antagonistiche, castratorie, parricide)» (Zatti 20I6: 296), in modo diametralmente opposto Mari sembra guardare invece alla mappa cartografica della tradizione nei termini di un gigantesco lascito da fare oggetto di una devozione mai estenuata (o solo pallidamente archeologica): gli scrittori del passato sono «i pionieri che hanno esteso la frontiera, che hanno combattuto, che hanno dissodato e arato ed edificato: i "padri". Quale scrittore, pensando alla letteratura, si dice più "Questa è la mia terra, e la difendo coi denti?"» (Mari 20I7: 733).

Deliberatamente polemica, la formulazione suona come un severo giudizio complessivo sul presente stato della narrativa italiana all'altezza dello snodo fra vecchio e nuovo secolo:

Da una quarantina d'anni il senso della tradizione è quasi azzerato non solo fra i giovanissimi, ma anche fra i meno giovani, per quanti libri possano aver letto (o scritto). Si è ormai radicata l'idea, vera solo in piccola parte, che la lingua e la letteratura crescano (per non dire nascano) insieme a una nuova percezione del mondo, e tanto più valgano quanto più abbiano la stessa età biologica del soggetto che le riceve e le pratica. La tradizione insomma è «vecchia», qualcosa di ammirevole, sì, ma come si può ammirare una vecchia magione di campagna senza però volerci abitare, una residenza scomoda da raggiungere o troppo costosa da mantenere... (Mari 20I7: 725).

La tradizione è insomma pensabile, per lo scrittore milanese, come un'abitazione posta in prossimità di un margine dislocato (fuor di metafora: sempre «al limite estremo del dicibile»). Le due immagini coalizzate 
(la similitudine «casa», e il connotato metaforico «frontiera») si sommano e puntualmente convergono in effetti nell'invenzione romanzesca che regge l'impianto di un romanzo come Verderame (ambientato in una casa di villeggiatura a pochi chilometri dal confine italo-svizzero). Ma quale idea Mari nutre, concretamente, della tradizione? Un'immagine polimorfa, inclusiva, enciclopedica: trovandosi essa a coincidere con «tutta la letteratura da Jacopo da Lentini e da Sordello in poi (questo per l'Italia, ma il principio vale per il mondo)» (Mari 20I7: 733). E l'immagine del perfetto scrittore (il talento che necessariamente governa la condotta di un «vero scrittore» nella contemporaneità) corrisponde per Mari all'identikit di qualcuno che sia in grado di muoversi «con familiarità lungo l'intero asse diacronico della nostra lingua, restituendo a vita una folla di parole e di modi sintattici che le ragioni dell'economia e della forza (cioè le ragioni meno letterarie che si possano immaginare) hanno da tempo condannato a morte» (694). In questi termini di partecipe ammirazione, il romanziere di Verderame parlava una volta di Gesualdo Bufalino: gettando un ponte, in modo esplicito, fra l'umbratile gusto antiquario del celebre autore de Le menzogne della notte e la terna di prosatori che per Mari rappresenta, entro il canone dei valori suoi personali, «il meglio del Novecento italiano» (694) - Tommaso Landolfi, Carlo Emilio Gadda, Giorgio Manganelli -, tre grandi scrittori accomunabili nel segno di una loro condivisa e segreta

\begin{abstract}
preoccupazione di inattualità, la quale come induce alla lingua una patina antica e arcaismi tanto significativi quanto obsoleti (pointe di una concezione antirealistica e antipedagogica della letteratura), così suscita di testo in testo dimore che sono anche e soprattutto una poetica: case nobili disfunzionali nella loro vastità come un Tommaseo-Bellini e pateticamente eloquenti nel loro stato d'abbandono (Mari 20I7: 205).
\end{abstract}

Segnatamente applicato a un ragionamento su Landolfi, il discorso consuona a ben vedere in toto con un passaggio del lungo e partecipe saggio che Andrea Zanzotto aveva dedicato (nel 1990) a una moderna riedizione de La pietra lunare (uno splendido intervento zanzottiano che Mari dovette al tempo della prima uscita leggere e recepire in termini di marcata consentaneità):

Come tutti coloro che cozzano contro le barriere del dicibile, egli [Landolfi] osa dei neologismi all'atto stesso in cui si compiace di riconoscerli come repêchages. E come «ripescante» egli è impagabile non meno che come inventore. In realtà egli attua tutta una sottile manipolazione di specchi tra neologismo, resurrezione dell'obsolescenza e anche ritmi entro i quali questo stesso gioco vien fatto, proprio come quando ci si mette alla roulette: tutto il 
vocabolario, in primo luogo, deve essere sfidato e messo alla prova su questa roulette (Zanzotto 200I: 333).

Dietro questa sostanziale riduzione della parola a fantasma (e dell'intero scaffale di tutti i vocabolari a una specie di museo d'ombre, per dirla con un titolo celebre di Bufalino), è facile sospettare la presenza fondamentale e per ogni verso decisiva del milanese Gadda, con la sua peculiare «méfiance gnoséologique à l'égard du discours ou plus précisément des mots» (Palmieri 20I2: 206). E nel milanese Gadda (concittadino di Mari) la divaricazione massima fra spinte linguistiche divergenti - producendosi nell'urto stupefacente e pirotecnico fra molteplici idioletti e una «componente fonica che spesso determina la scelta del vocabolo in ordine a una almeno apparente autonomia del significante» (Zublena 2000: 47 ) - ripropone in termini di espressionistica violenza un assunto che Mari raccoglie e fa proprio in sede storiografica, ragionando in materia di manierismo cinquecentesco, fra le pagine del Giordano Bruno degli Eroici furori: nella prosa di Bruno «il senso della continuità fra l'alto e il basso (o fra nobiltà e miseria, o fra menzogna e verità) svela che non c'è realtà astratta o concreta che non sia la forma provvisoria di un'indifferenziata energia» (Mari 2017:37).

Il corollario fondamentale che a questa premessa si appoggia è una concezione della lingua in Mari strutturalmente estranea a qualunque sclerotizzazione regolistica o pretesa normativizzante. Sentendo la lingua come un organismo divaricato nel tempo e nello spazio, lo scrittore-studioso milanese avverte il fascino dell'intarsio cosmopolitico di gerghi, dialetti e pronunce quale un necessario ingrediente che reinnesta, nella costitutiva arbitrarietà del segno linguistico, la fibrillante e caotica verità del mondo (con la sua musica sublime ovvero cacofonica, testimoniabile esemplarmente - fuori dall'ambito linguistico italiano - anzitutto nell'inarrivabile mistura della petite musique attuata dai romanzi di Céline, scrittore sempre molto diletto da Mari, sulla scorta di un'ardita riconversione e fusione di argot e francese accademico nella prospettiva di un lunare e depistante falsetto). Dislocandosi verso l'alto (nella zona sublime e parodica del calco dotto e libresco) o viceversa precipitando a picco nella parcellizzazione di un tendenziale indistinto borborigmo (comprensivo di inserti scurrili, invettivali, basso-mimetici), la concezione stilistica di Mari oppone, all'evanescenza potenzialmente anodina e insulsa di ogni mediana lingua standard, l'ideale di un'inappagabile «oltranza stilistica» (Mari 2017:38) che operi mettendo in sorvegliata giustapposizione segmenti eterogenei del linguaggio, continuamente sottoposti a una specie di programmatico urto calcolato.

«Non ci allontaneremmo molto dal vero - afferma lo scrittore nel citato Beneficio dellinfluenza - asserendo che Poe, Stevenson, Melville, Conrad e London si siano divertiti e appassionati a scrivere romanzi marinareschi 
proprio perché il filone già esisteva, ed abbiano provato il piacere di essere una perla della collana» (Mari 20I7: 730). E che la macchina romanzesca di Verderame si configuri segnatamente come un esplicito appassionato omaggio all'Isola del tesoro di Robert Louis Stevenson ${ }^{1}-$ del resto scrittore lungamente «ossessionato [...] dalla percezione di sé come doppio» (Mari 20I7: 94) - è evidenza che si dichiara a più riprese per tutto il romanzo: sino al conclusivo richiudersi della vicenda di Verderame sopra se stessa, ricomposta infine nei termini di una specie di ironico attraversamento di una linea d’ombra che riporta il narratore Michelino tredicenne alla normalità feriale delle sue giornate di annoiato villeggiante rampollo di borghesi:

\begin{abstract}
Forse il male non era così fascinoso come avevo sempre voluto, forse non era fatto di demoni e mostri... E anche l'avventura, che riuscivo a concepire soltanto nei modi stabiliti da Stevenson, da Melville e dagli altri numi che mi prendevano per mano, anche l'avventura forse era finita con loro, e solo ne rimaneva una squallida caricatura nella «Tv dei ragazzi», in certi orribili telefilm come I ragazzi di padre Tobia, che mi procuravano un immediato malessere già a partire dalla faccia di Silvano Tranquilli... (Mari 2007: 160).
\end{abstract}

Senza quel lievito potente e leopardiano che per Michelino è rappresentato dalla noia delle sue giornate agostane solitarie, nella ricca casa di villeggiatura dei nonni sul Lago Maggiore, la vicenda di Verderame non andrebbe nemmeno a incominciare: metaromanzescamente, la miccia d'innesco della narrazione (ambientata in un remoto anno 1969) è il desiderio di evasione implicito nella privata confabulazione di Michelino che inizia ad inventare (e prende a raccontare di fronte a se stesso) una storia romanzesca e intricatissima sui novecenteschi e gotici segreti riposti nelle mura della casa di Nasca (a cominciare dal passato dei suoi abitatori, primo fra tutti il giardiniere Felice). «Forse ero ingiusto io a vedere nel Varesotto il punto più angosciante del pianeta Terra» concede la voce adulta dello scrittore (Mari 2007: 87), mediando il ricordo e le riflessioni del suo trascorso alter ego tredicenne: ma il fatto è che quel lacustre paesaggio idillico, inscritto nell'orizzonte di giornate che il ragazzino riempie di mille curiosità fantastiche (al fuoco di enciclopediche letture), finisce per negarsi ad ogni sviluppo indirizzato verso la scoperta o l'avventura estranea alla mediazione libresca. Costretto a una specie di reclusiva inazione, dentro un hortus conclusus denegato all'avventura e sottoposto semmai all'ipoteca di una specie di suo melenso e soporifero surrogato (gli sceneggiati televisivi che inchiodano al teleschermo, tutte le sere, i nonni padroni di casa), il tredicenne Michelino si inventa la storia di Verderame e la minuziosa trama-diegesi del romanzo

I Mette conto ricordare che, su The treasure island, Mari si cimenterà qualche anno più tardi in un'impegnativa e felice traduzione d'autore (Stevenson 2015). 
come per tenere compagnia a se stesso (ricamando passo a passo, nel piacere di fabbricarsi in perfetta autonomia un organismo-centone, una specie di sperimentazione in vitro sul rapporto tra la fantasia e il mondo, i nomi e i realia, la compresenza di consequenzialità logica e delirio farneticante nell'attività di attribuzione dei significati ai singoli referenti oggettuali).

Quella sera, mentre i nonni vedevano uno sceneggiato con Aroldo Tieri e Sergio Fantoni, presi un cartoncino e scrissi in ordine alfabetico gli elementi principali di tutta la storia come fossero tarocchi. [...] Ricapitolai i punti salienti della storia dividendoli secondo verosimiglianza e inverosimiglianza, probabilità e improbabilità; separai ciò di cui ero stato testimone da ciò che mi era solo stato raccontato; individuai contraddizioni e aporie; stabilii una tavola di corrispondenze fra le trasfigurazioni del Felice e i fatti che dovevano averle provocate; sospesi in una zona limbica tutti gli elementi su cui era impossibile sapere qualcosa... Eppure, contro quell'intenzione positiva agiva in me uno spirito di decadenza e di abbandono che mi faceva privilegiare proprio le cose più assurde e misteriose (Mari 2007: I03-IO4).

Di questo valore doppiamente risarcitivo della storia raccontata (nei termini di una doppia compensazione, rispetto alla noia prodotta dalla fondamentale abulia dei taciturni avi borghesi, cosiccome davanti alla mancanza di energia affabulatoria veicolata dalle storie rappresentate sul teleschermo), il romanzo distribuirà del resto segnali indicatori a più riprese:

Probabilmente ero accoccolato di fianco a un povero diavolo afflitto da demenza senile, ma qualcosa, qualcosa che mi rifiutavo di attribuire a tutte le storie di pellerossa che avevo visto al cinema, mi persuadeva di essere a colloquio con un saggio o un veggente (40).

Tutte le combinazioni erano possibili, bastava sbizzarrirsi con la mente e qualsiasi risultato aveva una sua fantastica plausibilità: tanto più fantastica, tanto più plausibile. Hoffmann scriveva così: non a caso fin da allora lo ritenevo uno dei più grandi scrittori del mondo (64).

Io non ero uno scienziato che indaga per amore della verità: ero un esteta in erba che indagava per amore del brivido e dell'effetto, e in mancanza di tornaconto me ne sarei tornato in biblioteca a fare la vita di sempre... (66).

Io, piuttosto, dovevo incominciare a stare attento a trattenere in una sinossi mentale tutti gli elementi della storia, dai fatti accertati ai racconti più o meno verosimili del Felice alle mie ipotesi, perché solo tenendo tutto insieme potevo sperare di sceverare il falso dal vero e di arrivare a qualche conclusione decente (I2I). 
Idealmente, Michelino esce annoiato in giardino e fa mostra di concentrarsi sulla prima scena che gli appare alla vista, l'uccisione di una lumaca da parte del giardiniere di casa: prendendo le mosse da una descrizione che reca il segno della stravaganza, il romanzo può cominciare. Tuttavia, il registro architettonico della pagina incipitale di Verderame sposta immediatamente il piacere della parodia e dell'imitazione «à la manière de» in una direzione che non coincide in toto con il continente del romanzo avventuroso di tradizione anglofona. A tenere il libro a battesimo, in filigrana, il lettore avvertito non fatica a riconoscere il fantasma percepibile di Carlo Emilio Gadda; la qual cosa consuona del resto pienamente con un giudizio che lo studioso condensa altrove (Mari 20I7: 283) in forma di splendido epifonema («come Don Chisciotte, Gadda affronta sempre la realtà, siano otri o mulini a vento, con il massimo della serietà, quella serietà imbronciata del bambino che si concentra»):

Dimidiata da un colpo preciso di vanga, la lumaca si contorceva ancora un attimo: poi stava. Tutto il vischioso lucore le rimaneva dietro, perché la scissione presentava una superficie asciutta e compatta che il colore viola-marrone assimilava al taglio di una bresaola in miniatura. Dunque della sua bavosa vergogna l'animale si doveva liberare in continuazione per rimanere puro nell'intimo suo, e a questa nobile pena era premio la metamorfosi dell'immonda deiezione in splendida scaglia iridescente.

Corrugato da solchi paralleli e regolari, il tegumento esterno era di un rossiccio che teneva del boleto: ciò che distingueva il nostro mollusco come lumaca rossa ovvero lumaca francese: più tozza e più chiara delle nostrali, con una sagoma più vicina alla balena che al serpente, e corna più corte e meno facili alla protrusione.

- Puàh! - fece il villano sputando sopra il cadaverino ma mancandolo di qualche centimetro. Poi ritrasse la vanga e ne passò la lama fra due dita, come a nettarla di una poltiglia che esisteva solo nella sua testa. - Lümàgh frances! - e nuovamente esplose un bolo di saliva che come il precedente scaracchio nessuna benedizione avrebbe trasformato in madreperla. Lümàgh schifús vacaboia! - e finalmente si allontanava (Mari 2007:3).

La pagina si configura come un trasparente invito alla complicità ermeneutica del lettore di Mari, chiamato a riconoscere la natura del palinsesto implicato, e più ancora ad interrogarsi sul senso di questa inopinata irruzione del letteratissimo Gadda dentro il clima stevensoniano del romanzo. E un lettore che (accettando implicitamente questa sfida) intenda fare appello al deposito della propria memoria, non tarderà a ritrovare - nel racconto eponimo che in Gadda sigilla L'Adalgisa - il precedente che funziona da impronta per l'incipit-calco di Verderame (a partire dalla mania entomologica che prende il ragionier Carlo Biandronni e la passione di quest'ultimo per scarabei e coleotteri): 
Sul fronte del nerissimo insetto il ragioniere, felice, riconobbe l'epistòma, cioè la potente pala dentata, quasi uno spazzaneve di locomotiva. Dopo un po', vedendo che non succedeva nulla di nuovo, quella brutta bestia riprese la fatica. Puntava sulle zampe anteriori e retrocedeva in una sicurezza perfetta, come se ci vedesse dal pigidio. Ogni volta bisognasse afferrava la pallottola con le posteriori ed ecco, ecco la sospingeva all'insù, terribilmente, valicando con la tenacia di Sisifo le piccole dune, le increspature dell'arena [...].

E la sfera ascendeva, lenta: si sublimava sopra la repulsione di quella pazienza color pece, superava i tenebrosi divieti della gravità. Trasgredito il vertice, ripiombava rotolando nella gravità. L'Ateuco, infaticato, la sospingeva per monte e per valle fino alla dimora di sua donna: che attendeva, ansiosa, per il piccolo, per la imminente larva, quella balia provvidenziale.

[...] Carlo, tutto chino, con un batticuore, aveva già estratto le pinze. Con quelle afferrò l'Ateuco mentre si dibatteva furente, lo rinchiuse nel vasetto... Un ragazzo prese invece la pallottola, eccitato a conquistar la sua parte di fortuna; che però la sentì molle e anzi gli si spiaccicò fra i diti: «Ma un vedi che la è cacca, ettù bischero!...», strillarono ridendo i compagni. Quello rimase esterrefatto, con la polpetta stiacciata fra i quattro diti: poi corse alla battìma dicendo madonnabona madonnabona ziohàne (Gadda 20I2: 278279).

Su un piano linguistico, in entrambi i due passi messi a confronto, la severa e intonata compostezza della descrizione d'avvio - aulicamente intarsiata di arcaismi culti, forme latineggianti, frammenti di nomenclatura tecnico-scientifica - precipita nella reboante esplosione basso-corporea di una dialettalità che assume in Gadda, in prospettiva comico-grottesca, una inflessione caricaturale (un fiorentino dell'uso vivo - per così dire -, scimmiottato ad orecchio da uno scrittore non-toscano che lo incastona en abîme nella più vasta operazione satirica di cui fa oggetto e bersaglio i personaggi dei suoi disegni milanesi). Se il dialetto entra nel passo dell'Adalgisa come un mobile contrappunto della gaddiana prosa ipercolta (oppure riesce confinato topograficamente all'esplosione di un fuoco d'artificio in clausola), Mari riscalda invece l'elemento dialettale di Verderame spingendosi a un passo diverso e ulteriore. La radicalità, l'azzardo inusitato del libro è in sostanza quello di far parlare il doppio fantastico del tredicenne Michelino - ovvero l'illetterato giardiniere Felice - nell'arcigno dialetto della frontiera fra il varesotto e la sponda elvetica del Lago Maggiore.

Il dialetto di Felice è l'alterità assoluta (morfologica, culturale, geografica) rispetto alla norma toscana codificata. E Verderame scommette sulla pazienza di un lettore che - ragionevolmente estraneo ad ogni consuetudine extra-filologica con quel tipo di angusto veicolo comunicativo - non perda il passo della narrazione romanzesca ogni volta che il dialogo, fra Micheli- 
no e Felice, incappa nell'ostacolo di una formulazione dialettale in qualche modo impervia:

Fà la legna per i camin, dà de magnà ai gaijn, purtà el fen de sora, cambià i bumbul del Pibigas, fa la vendemia, strapà i erbasc, giüstà i erbasc, fa la calcina, fa 'l verderam... (Mari 2007: 30).

Beninteso, la pasta dialettale alto-lombarda che Mari compone e amalgama per dare voce al suo domestico mostro Felice risente calcolatamente di quel decisivo e onnipervasivo fenomeno, «l'italianizzazione dei dialetti», che caratterizza in generale il modo in cui lingua e dialetti «si scontrano e si intrecciano nell'Italia linguistica degli ultimi decenni» (De Mauro 20I4: I20). Eppure, alla consapevolezza esatta - che l'erudito professor Mari custodisce $a b$ origine nei suoi studi - di una diramata e reciproca "porosità» (Testa 20I4: I2) nell'articolazione degli scambi linguistici fra parlanti colti italòfoni e semicolti dialettòfoni, Verderame sostituisce il restauro ironico di una secca e più antica polarizzazione: unicamente in dialetto si esprime Felice, perlomeno sino alla pagina conclusiva del romanzo, nella quale il «ventriloquismo psichico» (Mari 2007: 132) che aveva sibillinamente presieduto alla trama del dialogo fra l'uomo e l'adolescente - «io pensavo e lui dava corpo sonoro al mio pensiero", aveva osservato a un certo punto Michelino (132) - precipita nei termini di una finale agnizione in restaurata lingua italiana. Michelino - confessa a quel punto Felice - «se vuole mi fa parlare tutte le lingue del mondo» (I64).

Ma quant'era brutto, quell'uomo? Provavo a immaginarmelo senza crateri vaiolosi, senza macchie e senza porri, senza gromma alle ciglia e con un naso meno sbrozzoluto e spugnoso, ma sempre brutto restava. C'era qualcosa di informe nel suo viso, come fosse stato modellato frettolosamente con il pongo: la bocca, in particolare, sembrava una ferita senza labbra, labbra che a ben vedere dovevano la loro ambigua percettibilità solo al colore, a quel violaceo che sembrava aggiunto come un rossetto. E poi c'era quella cicatrice verticale che scendeva da un occhio alla bocca, e che quando rideva si spiegazzava tutta... Già, non gli avevo mai chiesto come se la fosse procurata. Andai nella legnaia per chiederglielo ma vidi una cosa che mi fece dimenticare della mia domanda. Curvo sulla vasca, stava diluendo nell'acqua la pasta di verderame mescolandola con un palo (Mari 2007: I28).

Un raccapricciante e mite vecchione-bambino che racconta, per brandelli e gutturali lacerti, una misteriosa e intricata vicenda, attraverso la quale filtrano e si trasfigurano - in forma di complicato giallo familiare - le vicende tragiche della grande storia novecentesca (dagli echi della Rivoluzione sovietica all'epopea della Resistenza italiana). Lattempato Felice che 
non sa fare discorsi in lingua, e che è un fantasma d'uomo minacciato dalla prospettiva di un' incipiente demenza - «El mè nomm sacrabissa, come diaul me ciami, mi?» (Mari 2007: I0) - riesce agli occhi del tredicenne interlocutore (sotto la sferza del segreto bulino intrinseco alla sua fervida fantasia metaromanzesca) «un affabulatore maestoso», quasi «lo spirito di scrittori come Hoffmann, Poe e Lovecraft» rivivesse «in lui, l'uomo del verderame...» (52). Ora, il vecchione per antonomasia - nel paesaggio letterario del Novecento italiano - è senzameno Giuseppe Ungaretti, proverbialmente tormentato nella sua orfica lunga battaglia di un'intera vita contro l'inattingibile assillo di un'ancestrale «memoria demente» (Ungaretti 1969: I86). E il poeta di Sentimento del tempo - se riapriamo ora una specie di testimonianza-digressione (introducendo a un ragionamento critico di Mari sull'Odissea di Ippolito Pindemonte, raccolto ne I demoni e la pasta sfoglia) - sembrava intersecare decisivamente (apparendo nell'ipnotica veste di un indecifrabile e ironico aedo-affabulatore) lo spazio domestico delle avventure consegnate, in quell'Italia del 1969, alla fiction per il teleschermo in bianco e nero:

Per molti italiani l'incontro con Omero risale al 1969. Quell'anno, per diverse puntate, la RAI mandò in onda quello che con il Pinocchio di Comencini resta probabilmente il suo sceneggiato più bello: l'Odissea di Franco Rossi. [...] Eppure, di quelle serate, chiunque oggi abbia almeno cinquant'anni ricorda soprattutto una cosa, una cosa che era fuori dello sceneggiato vero e proprio: la performance di Giuseppe Ungaretti. All'inizio di ogni puntata, inquadrato in primo piano, Ungaretti recitava dei versi del poema: in parte a riassunto della puntata precedente, in parte ad anticipazione di quella che stava per incominciare. Intimidito e affascinato, il pubblico prendeva quei versi come una traduzione fedele, non necessariamente dello stesso Ungaretti: in realtà il poeta traduceva e inventava, contaminava, poetava à la manière de. Del resto come sottoporre a vaglio critico l'intervento di chi già nell'aspetto si presentava come un vate-sciamano, tutto scarruffato, con gli occhi semichiusi e la voce tanto arrochita e stentata quanto perentoria? È indubbio che nella coscienza dei più si creasse un cortocircuito fra la figura del poeta moderno e quella del poeta antico; per questo quelle introduzioni erano così emozionanti, perché era come se Omero entrasse in casa tua (Mari 20I7: 487)2.

Veggenti e mostri non possono che esprimersi, necessariamente, sempre in una lingua totalmente altra: donde l'impossibilità fattuale, per il vecchio,

\footnotetext{
2 Sulla poderosa valenza fantastica dei preamboli ungarettiani, riesce consonante anche la testimonianza di Boitani (2007: 42): «La RAI trasmetteva allora uno sceneggiato di Franco Rossi sull'Odissea, con Bekim Femiu e Irene Papas. Ogni puntata era preceduta da un breve intervento di Giuseppe Ungaretti, che declamava alcuni versi del poema, roteando la voce impastata e stentorea in guizzi improvvisi del capo e pause inattese. Mi è rimasta nelle orecchie l'eco - certo infedele filologicamente - di "Ulisse / uscìiva // sàlso // dal mare". Ungaretti era Demodoco. [...] Era Odisseo; era Omero».
} 
di attingere concrezioni di fraseologia banale nel suo tentativo di rispondere agli interrogativi di Michelino («La Rüssia? L’è un urs, el grand urs rüss. [...] il mio mostro rustico, parlare come un giornalista! Avesse anche aggiunto il Generale Inverno gli avrei tolto il saluto»; Mari 2007: 74). Il mite orco Felice è una trasfigurazione affettuosa e parodicamente stravolta di un Ungaretti camuffato? In questa luce, non sembra indebito leggere in chiave di parafrasi esilarante - rispetto al grande tema ungarettiano della «memoria demente» - il grottesco accesso di insofferenza che Mari attribuisce alla stanchezza di Felice assillato da Michelino: «Sun stracch, ghe ne podi pü de sta storia... podi no restà sansa memoria e vadavialcü?» (42). Eppure, portando ironicamente alle estreme conseguenze una premessa implicita nella poetica ungarettiana, l'equiparazione dell'analfabeta al veggente trova subito formulazione, in chiave di indizio metaromanzesco, nel gesto sapienziale e incantatorio con il quale Felice - all'inizio del libro - procede alla preparazione del verderame che darà il titolo al romanzo:

Sciolto nell'acqua, il verderame formava una pasta densa, simile a quella che nelle fiere di una volta i caramellai torcevano come lottassero contro un pitone: così doveva rimanere alcuni giorni per «respirare», verbo che diceva fin troppo bene della vita di quella cosa. A tal fine, il bidone restava pericolosamente aperto: io entravo più volte nella legnaia per controllare quella misteriosa attività respiratoria, e contemplando il meraviglioso turchese cercavo di non sporgermici sopra per paura delle esalazioni, una paura che mi confermavano gli insettini morti che sempre più numerosi maculavano il colore (Mari 2007: 5).

Anche per la coscienza demiurgica del narratore adulto, sembra difficile dare conto di cosa possa «simboleggiare un'immagine che può simboleggiare cose tanto diverse» (46). Ma il verderame è forse la misteriosa condensazione simbolica di un ideale di linguaggio totalmente pregnante e nuovo, costruito mescolando - nel vortice di un'unica furiosa tensione polifonica e centrifuga - le opposte e convergenti tentazioni del dotto riuso iper-manieristico e della bruta inserzione di una dialettalità tendenzialmente eslege. $\mathrm{E}$ la dominante complessiva che ne deriva finisce per coincidere, sul piano stilistico dei risultati, con una specie di partitura decostruita che attinge una forma di sublime d'en bas, frammentandosi in un forsennato caleidoscopio di tessere micro-musicali, funzionali da ultimo per rendere ragione della propria stessa diegesi-invenzione in chiave di sinossi farneticante:

Se avevo indovinato, se i Kropoff erano in combutta con i tedeschi, significava che il patto conveniva ad entrambe le parti, zaristi e nazisti... I secondi non espropriavano o deportavano i primi, i primi aiutavano $\mathrm{i}$ secondi facendo la spia, fornendo un appoggio logistico... In tal caso era 
quasi certo che avessero avuto parte nell'eccidio dei francesi... [...] Attirati in una trappola con l'inganno, ecco, i russi avranno offerto rifugio a un drappello di soldati francesi, dopodiché avranno avvertito l'Oberführer delle ss... Immaginavo con orripilata voluttà la scena, i russi che sciorinano il loro fluente francese e offrono da bere e da mangiare, gli ammicchi, le battute contro i fottutissimi boches... Sono capaci di aver sciolto una droga nel vino, hanno cura di servirsi solo delle bottiglie contrassegnate, ad ogni bicchiere i francesi sempre più rincoglioniti, qualcuno già ronfa sul tavolo... Poi un segnale convenuto, l'arrivo delle ss, i francesi vengono portati in cantina, la maggior parte di loro non si regge sulle gambe e rotola per le scale... In cantina li spogliano completamente perché la terra non rilasci un giorno mostrine o piastrine, poi a uno a uno li ammazzano con un colpo alla testa, c'è chi lo prende alla nuca e chi lo prende alla tempia, solo pochi hanno l'energia per reagire ma vengono falciati da una raffica... Di sopra, intanto, i due vecchi sparecchiano, forse c'è anche il figlio con loro... Stanno recuperando dai piatti gli avanzi che possono venir buoni per un altro pasto quando l'Oberführer entra in cucina battendo i tacchi: pochi ordini secchi, seppellire in giardino, pulire cantina, bruciare vestiti, Schnell! Come, non rimane nessun tedesco ad aiutarli? L'Oberführer non risponde nemmeno, un altro sbatter di tacchi, dietrofront e via, sparito (Mari 2007: I4O-I4I).

In termini che sembrano rievocare per analogia le complesse ambivalenze sottese all'esperienza di un grande funambolo-stilista del nostro Ottocento, quel Vittorio Imbriani che incarna entro il continente scapigliato la più sconcertante ed esatta prefigurazione di una «funzione Gadda» (Contini I970: 4), anche nella prosa di Mari «gli eventi urtano continuamente con la designazione di essi in forme moltiplicate fino a tendere all'esaurimento», fino al punto da trasformare potenzialmente la storia in una labile e fantasmatica «prestazione da vocabolario» (Bàrberi Squarotti 2005: 248-249).

Eppure «vicinissimo a Gadda nella vocazione all'ibrido e al mescidato» - osserva Mari (20I7: 529), con l'aria di rivendicare un pezzo decisivo del proprio autoritratto - «Imbriani non è pago di dare frustate alla lingua: più radicalmente, egli aggredisce le stesse convenzioni narrative facendo esplodere dall'interno la macchina romanzesca». Questa insubordinazione reca il segno di un rifiuto che investe ad un tempo (come in Gadda) «la convenzionalità delle tecniche linguistiche» e la «negatività dei valori» veicolati attraverso quelle tecniche (Spera 1976: 30). Interrogato esplicitamente su Verderame, Mari ha ricondotto la genesi del proprio romanzo a un medesimo atteggiamento di estraneità nei confronti di un ethos civile e di una temperie politica:

La vicenda storica che coinvolge la seconda guerra mondiale, i partigiani, il fascismo, il nazismo, è entrata come ingrediente fantastico attraverso gli occhi di un ragazzino che era sempre vissuto (e questo ha abbastanza riscontri 
autobiografici) in una casa fuori del mondo, in una specie di microcosmo torpido, con giornate ripetitive, una grande noia, con la sublimazione dello studio, però senza amici, senza coetanei, senza espansioni vitali. Perciò quando ho dovuto complicare la vicenda relativa a questa anamnesi, a questo ritorno alle madri, a questa discesa a ritroso nel tempo seguendo i ricordi più o meno frammentari di Felice, tutto quello che faceva brodo, che veniva buono allo scopo, l'ho utilizzato: il romanzo gotico, il vampirismo, il sangue, Puškin, gli zaristi, la storia russa, il кGв. E quindi anche le vicende dei partigiani, che ho accolto avendole filtrate attraverso rappresentazioni mitiche quali si trovano nei libri di Fenoglio, di Calvino, di Pavese. [...] La presenza della storia in Verderame probabilmente è dovuta anche a un'inconscia polemica nei confronti del filisteismo varesotto che ho sempre sentito come una cappa. La storia è ambientata a pochi chilometri da Gemonio, cioè dal paese di Bossi. I miei nonni sono sempre stati dei benpensanti: mio nonno era medico, mia nonna una dama di San Vincenzo tutta chiesa e opere pie. Così tra il filisteismo dei vari borghesotti milanesi che si facevano le ville intorno al lago e l'atteggiamento dei miei nonni, ho sentito forse il bisogno di iniettare nel romanzo qualche dose di storia (Mazza Galanti 20II: I75-I76).

Strutturandosi in forma di polifonia dissonante (con una naturale vocazione al pastiche e all'intarsio dei dialetti), «l'insoddisfazione nei confronti della norma linguistica» rimanda anche nel romanziere di Verderame - come in qualunque altro «eversore e deformatore» di generi e abitudini codificate (Mari 20I7: 528) - a un tipo differente di inquietudine, che non attiene più al registro delle forme, quanto al desiderio agonistico di trasformare «lo strenuo esercizio dello stile» nelll'«equivalente espressivo di un profondo senso del reale» (Isella I984: 24). Un'ambivalenza paradossale, della quale parlava del resto anche uno dei massimi esploratori della condizione dialettale nel Novecento italiano, Pier Paolo Pasolini (ripercorrendo i propri esordi, al tempo de La meglio gioventù):

Come linguaggio speciale per la poesia io adottai il friulano, ed era l'esatto contrario di ogni tendenza al realismo. Era il massimo dell'irrealismo, il massimo dell'oscurità. Una volta stabilito, tuttavia, il contatto con il dialetto, questo ebbe effetti inevitabili, anche se in origine l'avevo scelto per ragioni puramente letterarie. Non appena l'ebbi adottato, mi resi conto di essere approdato a qualcosa di vivo, di reale, ciò che ebbe l'effetto di un boomerang (Pasolini I999: 1289).

Irrompendo nel corpo della lingua e facendo mostra di occuparne lo spazio, il dialetto finisce per testimoniare - nel disegno compositivo di Verderame - una volontà di contestazione permanente, che Mari risolve nel solco della grande lezione modernista (inaugurata da Joyce): cancellando ogni gerarchizzazione stilistica nel modo di verbalizzare il caos ridondante della storia e della vita. 


\section{Bibliografia}

Bàrberi Squarotti, Giorgio, «L'avventura nella parola: Imbriani», Ottocento ribelle, Albano-Roma, Anemone Purpurea, 2005.

Boitani, Piero, Sulle orme di Ulisse, Bologna, il Mulino, 2007.

Contini, Gianfranco, Varianti e altra linguistica (I938-I968), Torino, Einaudi, I970.

De Mauro, Tullio, Storia linguistica dell'Italia repubblicana dal 1946 ai nostri giorni, Roma-Bari, Laterza, 2014.

Gadda, Carlo Emilio, L'Adalgisa. Disegni milanesi, a cura di C. Vela, Milano, Adelphi, 2012.

Isella, Dante, I Lombardi in rivolta. Da Carlo Maria Maggi a Carlo Emilio Gadda, Torino, Einaudi, I984.

Mari, Michele, Verderame, Torino, Einaudi, 2007.

-. I demoni e la pasta sfoglia, Milano, il Saggiatore, 2017.

Mazza Galanti, Carlo, Michele Mari, Firenze, Cadmo, 201 .

Palmieri, Giovanni, «La polyphonie du bas de page: Carlo Emilio Gadda et ses notes», La notion de «mineur» entre littérature, art et politique, sous la direction de B. Rodriguez et C. Zekri, Préface de J. Bessière, Paris, Michel Houdiard, 2012.

Pasolini, Pier Paolo, «Pasolini su Pasolini. Conversazioni con Jon Halliday [I968-I97I]», Saggi sulla politica e sulla società, a cura di W. Siti e S. De Laude, Milano, Mondadori, I999.

Spera, Francesco, Il principio dell'antiletteratura. Dossi, Faldella, Imbriani, Napoli, Liguori, 1976.

Stevenson, Robert Louis, L’isola del tesoro, traduzione di M. Mari, Milano, Rizzoli, 2015.

Testa, Enrico, L'italiano nascosto. Una storia linguistica e culturale, Torino, Einaudi, 20I4.

Ungaretti, Giuseppe, Vita d'un uomo. Tutte le poesie, a cura di L. Piccioni, Milano, Mondadori, 1969.

Zanzotto, Andrea, Scritti sulla letteratura. II. Aure e disincanti nel Novecento letterario, a cura di G. M. Villalta, Milano, Mondadori, 200 I.

Zatti, Sergio, «Intertestualità», in Stefano Brugnolo - Davide Colussi - Sergio Zatti - Emanuele Zinato, La scrittura e il mondo. Teorie letterarie del Novecento, Roma, Carocci, 2016.

Zublena, Paolo, «La scienza del dolore. Il linguaggio tecnico-scientifico nel Gadda narratore», L'inquietante simmetria della lingua. Il linguaggio tecnico-scientifico nella narrativa italiana del Novecento, Alessandria, Edizioni dell'Orso, 2002. 